\title{
Decision making for a delivery robot through a fuzzy system
}

\author{
Murillo Rehder Batista ${ }^{1}$ \\ Roseli Aparecida Francelin Romero ${ }^{2}$
}

\begin{abstract}
One of the main objectives of robotic researches has been the use of autonomous agents to make "mundane" tasks. A typical task common in literature is fetching a document from an initial point local and deliver to a specified destination. In this work, a fuzzy system has been developed to manage a number of requests that a robot has to deliver, which are requested in real time. This system uses a set of fuzzy rules to define the priority of each request based on conditions such as the time limit to take the document from its source or the score acquired from a successful delivery. A modification of $\mathrm{A}^{*}$ algorithm to plan paths and the potential field technique to manage navigation are used to guide the robot in the environment. Three different sets of fuzzy rules have been tested in order to compare their results with the known algorithm, firstin first-out, to attend the requests. The Player/Stage simulator has been used to make the tests. The results show that such approach is effective, obtaining better results than the first-in first-out algorithm in all tests performed with the three different sets of fuzzy rules and their objectives.
\end{abstract}

\section{Introduction}

One of the main objectives of robotics is to develop autonomous service machines able to do various tasks. Successful works such as robot RHINO [4], which was capable of making the role of a guide to visitors of a museum, and the humanoid robot ASIMO [21], capable of walking in difficult terrain and running, have brought possibilities of the most diverse task-making agents. A task that is very common in robotics is the delivery of documents, that is, take a document from an initial point and deliver to a specified destination point. Simmons et al.[23] developed a system to do such task capable of receiving real-time requests online. Another system to do deliveries was proposed in [10], focusing on places with a large stream of people like schools and hospitals. Bianchi and Romero [3] used artificial neural networks to control a robot to deliver documents in buildings and research laboratories of ICMC-USP. In many situations, it is necessary to choose among many options which are given in a moment, often delaying others. The same is applied to task-making agents: in a given moment, some tasks may have a greater importance than others. Simmons et al.[23] use the non-linear solver PRODIGY[5], capable of associating priority to tasks and to handle the

\footnotetext{
${ }^{1}$ Instituto de Ciências Matemáticas e de Computação, USP, Caixa Postal 668

\{rehderbatista@gmail.com\}

${ }^{2}$ Instituto de Ciências Matemáticas e de Computação, USP, Caixa Postal 668

\{rafrance@icmc.usp.br\}
} 
possibility of combining deliveries. An approach to decision making, which considers vague concepts, is that one known by fuzzy systems [15], systems which use fuzzy logic [29] to deal with imprecise information. In robot soccer, such systems were used to choose among various behaviors that one would execute during a match [27]. Lee et al. [16] used a rule-based fuzzy system to coordinate decisions of a team of safety robots, considering limitations such as terrain coverage and remaining battery of the agents. Various works which take benefit from the rule-building nature of fuzzy systems are also found in applications ranging from topics as land suitability evaluation [19] to WiFi localization systems [1]. In this work, a fuzzy rule-based system is proposed to evaluate the priority of delivery requests realized by an autonomous robot, with the objective of verifying if such decision making method is adequate to handle this type of decision and if it is easy to use such structure to generate different patterns of decision making through expert knowledge. A test delivery system with path planning and navigation modules is built in order to test the proposed system. This system has been tested on simulation level through the Player/Stage[11] simulator, in which a Pioneer2x robot equipped with a laser rangefinder has been chosen. In order to make this task easier, it is considered that the act of "taking" or "delivering" a document is equivalent to reach its source or destination localizations. It is considered that an occupancy grid [8] was built before the system starts. The navigation system is necessary to verify how changes caused by recieving a new delivery request with greater priority might influence in the movements made by the robot. This paper is organized as it follows. In Section 2, a brief review of the concepts used is made. In Section 3, the proposed implementation is presented. In Section 4, the tests are explained and the obtained results are discussed. In Section 5, the conclusion is presented, along with future works.

\section{Review}

In this Section, the concepts and techniques which are used in this work are explained briefly. In Section 2.1, an explanation about path planning and navigation methods, namely the A* algorithm, the Potential Fields technique, and the Harmonic Potential Fields method. In Section 2.2, some fuzzy logic and fuzzy systems concepts are introduced.

\subsection{Path planning: the $A^{*}$ algorithm}

A common problem which appears often in Robotics is how to make a robot able to navigate in an environment, given or not a map. When it is considered that there are no dynamic objects and we have a map, a deliberative approach may be considered [17].

A classic technique that may be used in such case is the $A^{*}$ algorithm [12] [20], proposed to find an optimal path between two nodes of a graph. Uses and improvements 
of this algorithm are often found in various works, including path planning for groups of unmanned aerial vehicles [22], virtual human motion path planning [28] and path planning for mobile geographic information systems [25]. Such method starts its search from a starting node, inserting all its neighbor nodes into an "opened list", which represents candidate nodes to be verified in the next iteration, and the starting node into a "closed list", which will have nodes that have been already checked.

When a node is inserted in the "opened list", it is associated with a path cost and a link to its neighboring node which reached it. Such cost is the sum of its parent node's cost to an heuristic that estimates the cost to get to the destination node. This heuristic serves as a guiding parameter which directs the search towards the destination node. It is important to ensure that the estimative calculated by such heuristic is smaller than the actual cost if a strictly optimal path is wished. Otherwise, the search result may be non-optimal. When we set the heuristic value to 0, the search works exactly like Dijkstra's algorithm [7]. Such heuristic, however, results into losing the "guided search" aspect of the A* algorithm.

If a node which is to be inserted in the "opened list" is "closed", it is not inserted. In case such node is already "opened", its cost and link to its parent neighbor are updated if the new path's cost is smaller than the one already stored in that node. In the end of each iteration, the node from the "open list" with the smallest path cost is chosen to continue the search, becoming a "closed" node.

When the destination node is achieved, the optimum path is obtained through backtracking the neighbor links until the source node is found.

In many cases, however, there is not enough information about the environment to use an deliberative approach with effectiveness. In such scenario, some procedure based on the reactive paradigm [2] must be considered.

\subsection{Navigation through Potential Fields}

To manage navigation in conditions where data on the environment is not complete, a reactive technique based on the sum of forces known as Potential Field method was proposed [14]. This technique is applied to navigate in an environment exercising an attractive force towards a destination location and repulsive forces from nearby obstacles so the robot will be conducted by such forces until it reaches its destination.

The attractive force $\mathrm{F}_{a t}$ applied is obtained by the Equation 1], where $\mathrm{C}$ is a constant; Equation 2 shows how to obtain the repulsive forces $\mathrm{F}_{\text {re }}$, where $\mathrm{Q}$ is a constant, $\mathrm{d}$ is the Euclidean distance from the robot to the destination. In both equations, $\Delta \mathrm{x}$ and $\Delta \mathrm{y}$ are the distances corresponding to the $\mathrm{x}$ and $\mathrm{y}$ coordinates from the robot position to the destination 
position.

$$
\begin{gathered}
\left|F_{a t}\right|=C, \theta_{a t}=\arctan \left(\frac{\Delta y}{\Delta x}\right), \\
\left|F_{r e}\right|=\frac{Q}{d^{2}}, \theta_{r e}=\arctan \left(\frac{-\Delta y}{\Delta x}\right),
\end{gathered}
$$

According to Equation 2, nearby obstacles apply greater force than distant ones, which yield negligible effects. The attractive forces, however, always have the same module. Figure 1 illustrates the force vectors from many possible robot positions.

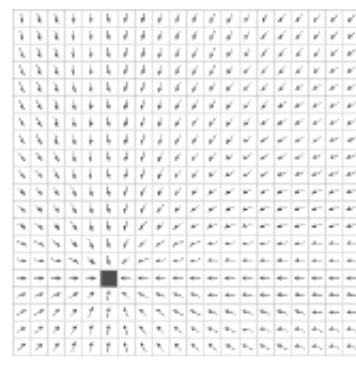

(a)

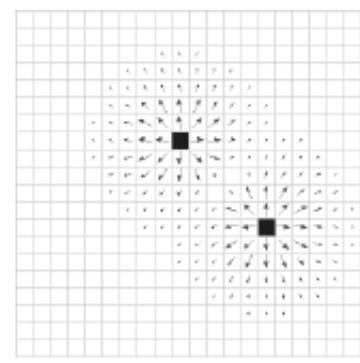

(b)

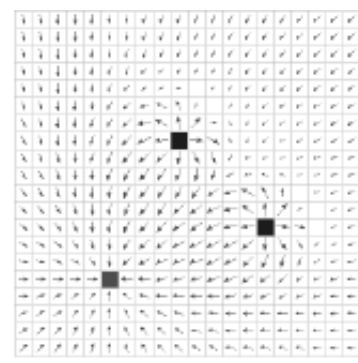

(c)

Figure 1. Figure 1(a): attractive forces towards a destination; Figure 1(b): repulsive forces starting from obstacles; Figure 1(c): Sum of the forces of Figures 1(a) and 1(b)[9].

Such method has many advantages, especially low computational cost and ease to deal with sensor readings. However, it suffers of local minima cases due to the nature of generation of forces (sum). Also, without the proper constant tuning, the robot might collide with obstacles near the destination position or become unable to reach it because of low attractive forces in comparison to repulsive ones. In many cases, especially the latter ones, a better adjust of the constants solve the problem.

In 1993, a new enhancement to Potential Fields was proposed, called Harmonic Potential fields [6]. This approach uses a solution to Laplace's equation described in Equation 3 to calculate forces instead of sums, ensuring that there will always have a path to the desired destination through the generated forces if such path exists and that no local minima appears. The value $p$ represents the potential force in a point of a continuous environment.

$$
\nabla^{2} p=\sum_{i=1}^{n} \frac{\partial p^{2}}{\partial x_{i}^{2}}=0
$$


As this equation has no analytical solution yet, a numerical resolution through a grid is adopted, transforming the problem in a solution given by Equation 4, where $p$ represents the potential force of a discretized grid-like potential grid.

$$
p_{i, j}=\frac{p_{i-1, j}+p_{i+1, j}+p_{i, j-1}+p_{i, j+1}}{4}
$$

To use such approach to navigate, it is considered that all cells in the grid with obstacles have value 1 and that the destination cell has the value 0 . The remaining grid positions are updated to solve Equation 4. An applicable solution is the Gauss-Seidel method [6], where the occupation grid is covered point-to-point continuously to update the forces. In Figure 2. forces generated by this method are shown.
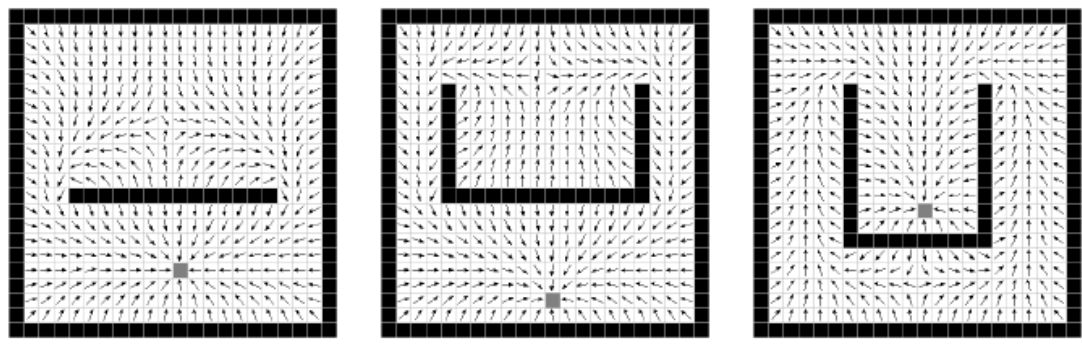

Figure 2. Repulsive forces in various environments 9 ].

This method has, however, a major drawback in its depedency on the occupation grid. When the grid is not fine grained enough, i.e., the cell's size is too big, the robot is prone to collide in minor details which were not covered by the map; very detailed grids, however, result in very high computational costs for a method which has to be executed often during navigation tasks.

\subsection{Fuzzy Logic and Fuzzy Systems}

In the decade of 60, an extension of boolean logic was developed, known as fuzzy logic [29]. While in boolean logic a statement is strictly true or false, fuzzy logic is able to deal with a set of membership degrees ranging from 0 to 1 . The nearer from 0 (false) or 1 (true) the degree is, the smaller the uncertainty is.

In Equations 5,6 and 7, the corresponding forms of union, intersection and negation are shown. Note that such operations can also be used for boolean logic, therefore being an extension of boolean logic operators. Like boolean logic, it is possible to work with sets 
within fuzzy logic, applying logic operations between fuzzy sets.

$$
\begin{gathered}
A(x) \cup B(x)=\max (A(x), B(x)) ; \\
A(x) \cap B(x)=\min (A(x), B(x)) ; \\
! A(x)=1-A(x) .
\end{gathered}
$$

An important concept is membership functions, which are a set of functions that associate a numerical value to linguistic variables. Through them, it is possible to do a fuzzification: the process of converting a crisp number into a set of membership degrees through membership functions. There are many types of membership functions that can be chosen to make associatons. In Figure 3, are shown the triangular, trapezoidal, gaussian and bell membership functions.

The reverse process, that is, the process of transforming various grades of membership into a crisp number, is called defuzzification. There are many techniques to do this conversion, such as the Center of Mass (CoM) method, which captures a centroid of the grades of membership, and the Maximum of Average, that obtains the medium value of the points with greater grades of membership [18].
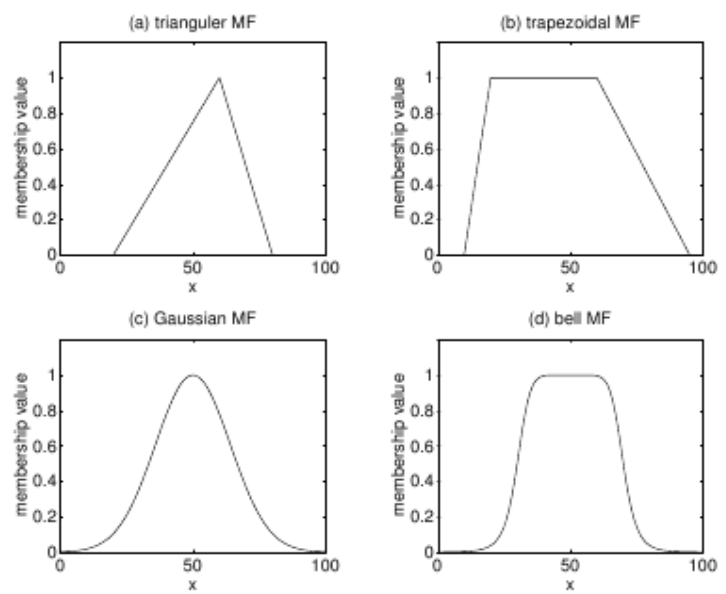

Figure 3. Membership functions. (a): triangular; (b): trapezoidal; (c): gaussian; (d): bell[13].

With such concepts, a rule-based system which uses fuzzy logic to make decisions, or Rule-based Fuzzy System (or fuzzy system) can be built. The most remarkable advantage 
of such system rely on the treatment of linguistic concepts, present in the spoken language, involved in the problem that one wishes to solve. The first step into making a fuzzy system is to define its inputs and outputs, as well as their universes of discourse, that is, the set of numerical values which will be associated with the linguistic labels. The next step is to elaborate the linguistic labels to each input and output. Then, it is needed to build a rule base that deals with the linguistic labels to associate input and output sets. Such rules are known as fuzzy inference rules. To analyze the grades of membership in relation to the rules, a fuzzy inference model is chosen: common choices are the max-min and the sum-product models [18].

Perhaps the most known fuzzy system is the Mamdani model [15]. In this model, inputs are fuzzificated to become membership degrees. The inputs are related to the outputs through the rule base. The result of such process is applied in a defuzzification method to achieve a crisp output. This model is known for being comfortable for an expert to insert knowledge obtained by experience.

Another common model is the TSK, or Sugeno model [24]. Its difference to the Mamdani model lies on association of the rule consequents: there is no association to a specific linguistic variable, but to a polynomial function. In this method, defuzzification occurs within inference itself. This model allows a greater freedom to apply optimization methods than the Mamdani model due to the use of polynomial functions at the cost of being less intuitive for the insertion of expert knowledge.

\section{Proposed robotic delivery system}

The robotic delivery system that is proposed in this paper is separated in four modules, described to follow:

Navigation Module: defines how the robot goes to somewhere considering obstacles in its path;

Path Planning Module: traces the route which is followed by the robot to reach a source or destination position for getting documents;

Decision Making Module: gives priority to delivery requests received;

Central Module: communicates with the robot and all other modules in order to manage the system.

The occupation grid that represents the environment is loaded and a priority queue which will store received requests is initialized. After this step, the main cycle, that is, the 
execution of the Central Module, starts. At any moment, the system might get a request. While no request is received, the robot stays idle. When a request is received, an ID is given to it, the Decision Making Module is activated to give a priority value to the request, and the request is put in the priority queue. If the robot is not delivering a document (i.e., it has already taken a document and is going towards its destination), a new task might be chosen. If a task is received and no other request is in the priority queue, the task is chosen. The request which has been selected is the one on top of the priority queue, being "popped" out from the queue when the request's document is taken to be delivered. When the robot is going towards the task's document source, a request with a greater priority might appear. In this case, the new request will naturally be on top of the priority queue, becoming the new task to be picked.

Every time a new request is chosen or the robot takes a document, the Path Planning Module traces the route to be followed through the previously read occupation grid. This module returns to the Central Module a series of sub-goals to be followed by the Navigation Module, that by its turn gives an angular speed to the Central Module. Using the laser sensor readings, the system defines the linear speed, which slows down the nearer it gets from a detected obstacle.

In the end of the iteration, all priority values are recalculated and inserted again into the priority queue. Recalculation is needed because of evaluating criteria that may vary over time, such as distance-based ones.

All the system was developed in $\mathrm{C}++$, and it was implemented to run on the Player/Stage simulator, that is, the Player interface, that has drives that communicate with the robot, combined with the Stage simulator [11]. In Sections 3.1 and 3.2, an explanation on how the concepts shown in this Section were used to, respectively, path planning/navigation and decision making.

\subsection{Path Planning and Navigation Modules}

As it was mentioned before, both Path Planning and Navigation Modules are responsible to make the robot go to a specific place in the environment. Initially, the Harmonic Potential Field method was used for both purposes using the Gauss-Seidel approach to update forces. However, there was no indication that an optimal path would be found. Given this issue, the Harmonic Potential Field was set only as a navigation method, following a trail of goals generated by the Path Planning Module. built with the $\mathrm{A}^{*}$ algorithm using a heuristic $\mathrm{H}$ given by the Equation 8 , which respects the optimum condition because it will always be smaller or equal to the actual traveled distance. After the path is traced, three-to-three grid cells from the generated path are given to the Central Module: these squares are the trail of 
sub-goals to be followed.

$$
H=\max (\Delta y, \Delta x)
$$

The generated path by $\mathrm{A}^{*}$ algorithm is, however, too close to walls. To avoid this, a modification was done on the cost calculation, multiplying a node cost by a $\mathrm{M}_{c}$ constant in case of the distance between the nearest walls on horizontal or vertical positions and the robot's position according to the occupation grid is too big. It was considered, too, a maximum number of grid positions $\operatorname{Max}_{P}$ to be checked around the robot. In spite of turning the path generated non-optimal, this modification enabled the robot to stay futher from walls while generating a good path. In Figure 4 , it is shown the difference between a path generated by $A^{*}$ techinque and a path generated by the proposed modification with $\mathrm{M}_{c}=-0.1$ and $\operatorname{Max}_{P}=3$.

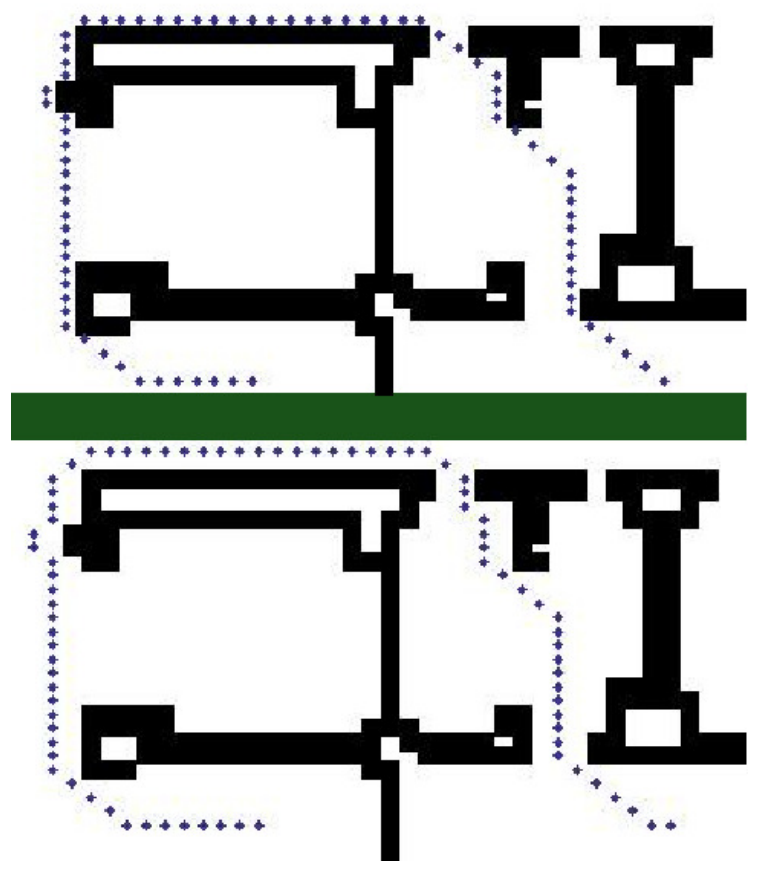

Figure 4. Path generated by A* method.

Following a trail of sub-goals that ensures an optimal path, the path planning issue was solved. However, the Harmonic Potential Field's effectiveness as a navigation method relies on a very fine grained representation of the environment: details not shown by the map 
might result in collisions. Considering that the distance between sub-goals is small and the linear speed is dependent only of the laser readings, chances of a local minima outcome for a traditional Potential Field technique are drastically reduced, so this method was tested as the Navigaton Module using only sensor information to detect obstacles. The more detailed detection of hurdles allowed a safer navigation. Figures 5 and 6 show the difference between, respectively, the use of Harmonic Potential Fields and Potential Fields following the A* trail. Notice that details unnoticed by the occupation grid resulted in collisions in Figure 5 The routes were taken during execution in the Player/Stage simulator.

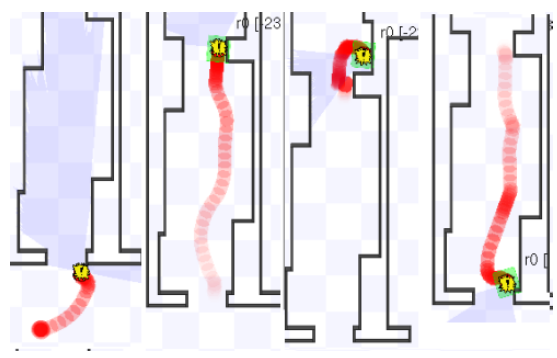

Figure 5. Navigation through Harmonic Potential Fields.

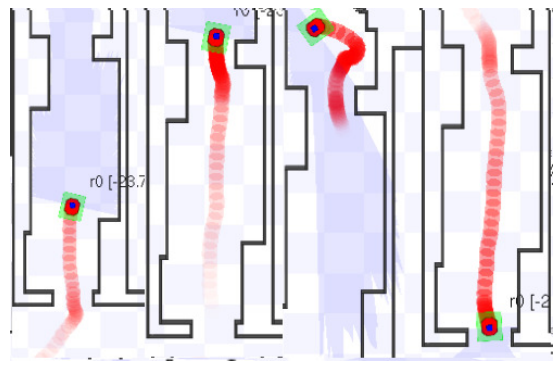

Figure 6. Navigation through Potential Fields and A*.

\subsection{Decision Making Module}

The objective of the Decision Making Module is to give priorities to delivery requests. This project's proposal is the use of a Mamdani-type fuzzy system to do this task because of its ease to insert expert knowledge. The developed system was made in Xfuzzy tool[26], which translated the formulated system into $\mathrm{C}$ code. The fuzzy system had three informations:

Dist: sum of the Euclidean distances robot to source of the document and source to destina- 
tion;

Time: maximum time to get a document in its source before the request is canceled (in seconds);

Score: score obtained on each succesful delivery. It may be seen as money paid for the service done.

These values are used to obtain the fuzzy system's inputs $\mathrm{P}$ Dist, $\mathrm{P}$ Time and $\mathrm{P}$ Score, shown in Equations 9, 10 and 11. CDist, CTime, and CScore are constants used to regulate value magnitudes and StartTime is the instant (in seconds) in which the Central Module started working since the request was received. The fuzzy system receives values between 0 and 1 as input parameters.

$$
\begin{gathered}
P_{\text {Dist }}=\max \left(\min \left(\frac{\text { Dist }}{C_{\text {Dist }}}, 1\right), 0\right) \\
P_{\text {Time }}=\max \left(\min \left(\frac{C_{\text {Time }}}{\text { Time }+ \text { StartTime }}, 1\right), 0\right) \\
P_{\text {Score }}=\max \left(\min \left(\frac{\text { Score }}{C_{\text {Score }}}, 1\right), 0\right)
\end{gathered}
$$

For the fuzzification, trapezoidal membership functions are used. They are displayed in Figure 8, and represent three linguistic values: "low", "moderate" and "high". In Figure 7 are shown the membership functions of the consequents of the rules, with "yes" and "no" as linguistic values. The adopted fuzzy inference method is max-min. Figure 8 corresponds to the membership functions of the output.

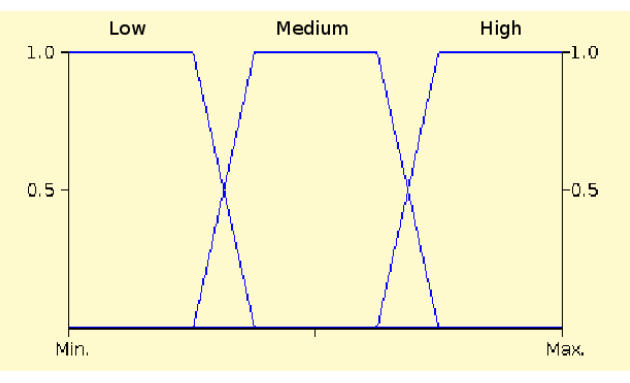

Figure 7. Membership functions of the input. Functions plotted by Xfuzzy [26]. 


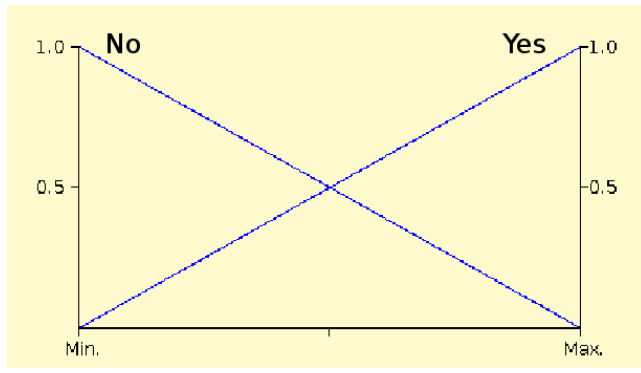

Figure 8. Membership functions of the output. Functions plotted by Xfuzzy [26].

Fuzzy inference rules are elaborated in the format of IF-THEN rules, such as:

IF $\mathrm{P}_{\text {Score }}=\mathrm{HIGH}$ AND $\mathrm{P}_{\text {Time }}=\mathrm{HIGH} \rightarrow$ OUTPUT $=$ YES $*(\mathrm{w})$.

It is possible to associate a weight $w$ to the influence of every rule of the system as numbers that multiply the output of each rule. These numbers range from 0 to 1 . Such resource is convenient when it is desired that some rules have a greater importance factor than others.

For defuzzification, the Mean of Maxima method is used, i.e., the greatest output value is chosen and an average value between the associated crisp outputs is obtained. This output ranges from 0 to 1 , and greater values indicate a greater priority for a document request.

\section{Tests}

To evaluate the proposed system, a path planning/navigation test was performed to verify its limitations and a decision making test was applied to test the fuzzy system as a decision making tool. These tests are made separately to avoid that a critical robot navigation (a collision) issue interferes with the analysis of the decision making process. Both tests were performed using the Player/Stage simulator, with a simulated Pioneer2x robot equipped with a $180^{\circ}$ wide SICK laser sensor. The adopted environment is a default $20 \times 15 \mathrm{~m}$ Player/Stage map called "889_05". For all tests, a sensor noise generator provided by the simulator was enabled.

\subsection{Path Planning and Navigation Tests}

Three different tests to evaluate the Path Planning and Navigation Modules have been performed. The maximum linear and angular speed achievable by the robot were respectively $0.5 \mathrm{~m} / \mathrm{s}$ and $\pi / 10 \mathrm{rad} / \mathrm{s}$. The combination Potential Fields with modified $\mathrm{A}^{*}$ algorithm was 
used, with $\mathrm{M}_{c}=-0.1$ and $\operatorname{Max}_{P}=3$.

In the first test for the navigation, a narrow corridor scenario was used. The robot had to go inside the room after the corridor and return to its starting point. In Figure 9 , different stages of the path taken by the robot are shown. The red trail let by the simulated robot in the images grow weaker as time passes. Some difficulty to go through the narrow corridor was detected, but no collisions occured.

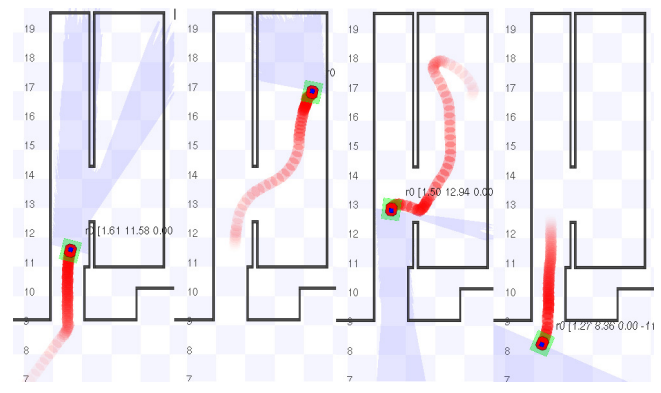

Figure 9. First path planning and navigation test.

The second test involved a room filled with agents performing a "wander" behavior and a door. The objective is making the robot pass through the door. Figure 10 shows the robot's trajectory: the alphabetical order indicates the sequence of the events. Despite the difficulty shown to dodge a wandering agent while reaching the door, the objective was achieved without any contact between the robot and an agent or a wall.

A mix of the previous test was made in the third test: the same place and objective of the first test, but with the agents of the second test. Figure 11 indicates the sequence of navigation. As it can be seen in the final quarter of the images, the robot was unable to dodge an agent which came in its direction, resulting in a collision.

Although the navigation and path planning approach attained its objective, some enhancements may be needed for this system be useful in real environments, especially because of the traffic of people and other potential dynamic obstacles.

\subsection{Decision Making Tests}

Using the same path planning and navigation configurations of previous tests, a delivery request scenario was built in order to test the proposed fuzzy system for decision making. Three different rule bases were developed and compared to the classic first-in-first-out (FIFO) approach, that is, always attending requests exclusively by order of arrival. The rule bases were built through interviews with 20 people, being 12 undergraduate students, 7 with high 


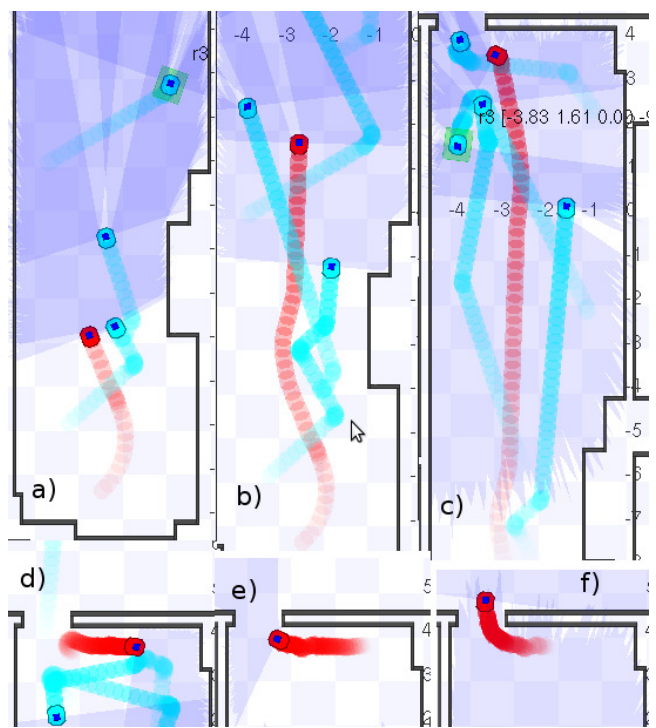

Figure 10. Second path planning and navigation test.

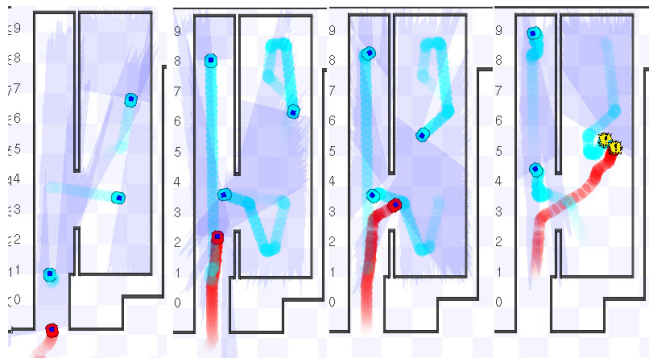

Figure 11. Third path planning and navigation test.

school completed but not into college, and one person that often deals with decision making of which task will be done on each day of work, therefore being the nearest of an "expert" to be interviewed. It was told them that the main focus was on attending the maximum requests possible, considering score being a secondary issue. None of the interviewed people had any contact with fuzzy systems before.

Rules were built after a brief explanation to the interviewed people on the approached problem and fuzzy systems, especially on the fuzzy rules structure. Based in such information and personal experience, two rule bases were built. A third rule base was built for result comparison. 11 out of 20 people were not able at all to understand how to build such rule 
bases thus their opinions were discarded.

The Urgency Model gives priority to tasks with a tighter time to be taken, leaving score and time as peripherical worries. The rule base of the corresponding model presented below has been built based on the opinions of some interviewed people. The value multiplicating the output is the weight $\mathrm{w}$ of the rule.

1) $\mathrm{IF}_{\text {Time }}==\mathrm{HIGH} \rightarrow$ OUTPUT $=\mathrm{YES}(* 1.0)$;

2) IF $\mathrm{P}_{\text {Time }}==\mathrm{LOW} \rightarrow$ OUTPUT $=\mathrm{NO}\left({ }^{*} 0.8\right)$;

3) IF $_{\text {Score }}==$ MEDIUM AND $\mathrm{P}_{\text {Dist }}==\mathrm{LOW} \rightarrow \mathrm{OUTPUT}=\mathrm{YES}(* 0.2)$;

4) IF $_{\text {Score }}==$ HIGH AND $\mathrm{P}_{\text {Dist }}==\operatorname{MEDIUM~} \rightarrow$ OUTPUT $=\mathrm{YES}(* 0.3)$;

5) IF $\mathrm{P}_{\text {Score }}==$ LOW OR $\mathrm{P}_{\text {Dist }}==\mathrm{HIGH} \rightarrow$ OUTPUT $=\mathrm{NO}(* 0.2)$.

The Distance Model was based on the "expert" information. This model emphasizes on attending nearest requests first, letting time and money on background. The corresponding rules are shown below.

1) IF $\mathrm{P}_{\text {Dist }}==\mathrm{LOW} \rightarrow$ OUTPUT $=\mathrm{YES}(* 1.0)$;

2) IF $P_{\text {Dist }}==\mathrm{HIGH} \rightarrow$ OUTPUT $=\mathrm{NO}(* 0.8)$;

3) IF $\mathrm{P}_{\text {Time }}==$ MEDIUM AND $\mathrm{P}_{\text {Score }}==\mathrm{LOW} \rightarrow$ OUTPUT $=\mathrm{YES}\left(*^{*} 0.2\right)$;

4) IF $\mathrm{P}_{\text {Time }}==\mathrm{HIGH}$ AND $\mathrm{P}_{\text {Score }}==\operatorname{MEDIUM} \rightarrow$ OUTPUT $=\mathrm{YES}(* 0.3)$;

5) $\mathrm{IF} \mathrm{P}_{\text {Time }}==\mathrm{LOW}$ OR $\mathrm{P}_{\text {Score }}==\mathrm{HIGH} \rightarrow$ OUTPUT $=\mathrm{NO}(* 0.2)$.

For comparison, a third model named Greedy Model was built based on the former models aiming its priority in score acheiving rather than number of deliveries done. The corresponding rules are shown below.

1) $\mathrm{IF} \mathrm{P}_{\text {Score }}==\mathrm{HIGH} \rightarrow$ OUTPUT $=\mathrm{YES}(* 1.0)$;

2) IF $\mathrm{P}_{\text {Score }}==\mathrm{LOW} \rightarrow$ OUTPUT $=\mathrm{NO}(* 0.8)$;

3) IF $\mathrm{P}_{\text {Time }}==$ MEDIUM AND $\mathrm{P}_{\text {Dist }}==\mathrm{LOW} \rightarrow$ OUTPUT $=\operatorname{YES}(* 0.2)$;

4) IF $\mathrm{P}_{\text {Time }}==\mathrm{HIGH}$ AND $\mathrm{P}_{\text {Dist }}==\operatorname{MEDIUM} \rightarrow$ OUTPUT $=\mathrm{YES}(* 0.3)$;

5) IF $\mathrm{P}_{\text {Time }}==$ LOW OR $\mathrm{P}_{\text {Dist }}==\mathrm{HIGH} \rightarrow$ OUTPUT $=\mathrm{NO}(* 0.2)$.

It can be seen that the weights of the rules are the defining characteristic that separates rules of different importance factors.

The decision making scenario was built choosing 44 locations of the environment, which are marked in Figure 12 with an $\times$. A total of 33 requests were built by randomly 
setting sources and destinations from the chosen locations. The other informations were, too, randomized: scores were taken between values 0 and 100, limit time between 0 and 2000 seconds, and time of request submission ranging between -500 and 2000 seconds, where values equal or less than 0 means that the request was performed when the Central Module just started to work. THe values given to CDist, CTime, and CScore are, respectively, 0.5, 0.5 and 0.1 , and were chosen observing that score values have a considerably shorter range in comparison to the other measures. In this test, the wandering agents were not used in order to avoid eventual collisions during a test designed to evaluate the performance of the decision making system. To simulate an incoming request, the system used a timer to count the time elapsed since the system starts to work: when the starting time of a request comes, it is received by the system.

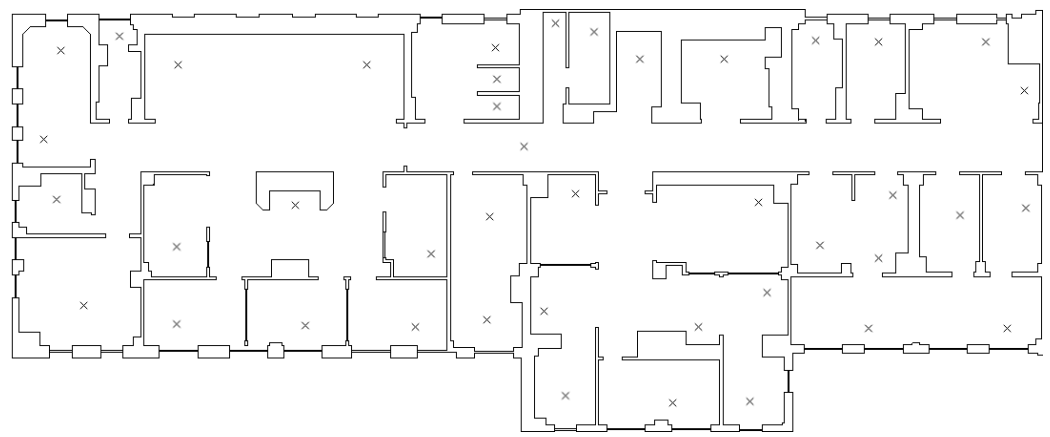

Figure 12. Key locations used for random allocation of sources and destinations of the requests.

In Table 1, the decision making test results are shown. During the tests, no collision incident happened. It is important to notice before analyzing the results that the built test scenario was intended to be impossible to achieve a rate of $100 \%$ requests done in order to test the system in conditions with many requests to be chosen. Execution time is shown in seconds. All models achieved better results in comparison to the FIFO approach, with the exception of the delivery rate of the Greedy model (33\% of the requests), which was not built with the intention of covering the greatest number of requests possible, focusing on scores. That model got, however, the best score. The rule base with the best request rate was the Distance Model, the one suggested by the "expert", attending $48 \%$ of the requests.

\section{Conclusion}

In this work a robotic delivery system able to receive requests in real time was proposed. This system uses a fuzzy inference system combined with a path planning made by a 
Table 1. Results of the Decision Making test.

\begin{tabular}{|c|c|c|c|}
\hline Model & \# of requests & Score & Time \\
\hline \hline Urgency & $14(42.4 \%)$ & 740 & 2449 \\
\hline Greedy & $11(33.3 \%)$ & 816 & 2318 \\
\hline Distance & $15(48.5 \%)$ & 787 & 2068 \\
\hline FIFO & $12(36.4 \%)$ & 566 & 2336 \\
\hline
\end{tabular}

modification of the $\mathrm{A}^{*}$ algorithm and a navigation managed by a potential field approach to attend the requests given by the user. Despite the limitations of the path planning and navigation techinques, they were efficient enough to realize the decision making tests, which was their main objective. The fuzzy system approach showed itself effective considering the good results obtained, especially when compared to the simple FIFO approach. In many cases, the fuzzy system delayed a chosen request when a more convenient one appeared, what was expected and worked well. The process of building fuzzy rules was comfortable to the "expert", but not to the people without experience with similar decision making situations or without prior knowledge of fuzzy systems.

As future work, it is suggested to build a complete delivery system, with mapping, localization, and an interface to insert the requests in order to test the proposed system in a real robot. Another suggestion is to use a team of robots to do deliveries. Another idea is to test new approaches on the fuzzy system, like different defuzzification techniques or the use of a Sugeno model, aiming the use of a learning method.

\section{References}

[1] J. M. Alonso, M. Ocaña, M. A. Sotelo, L. M. Bergasa, and L. Magdalena. Wifi localization system using fuzzy rule-based classification. In Computer Aided Systems Theory EUROCAST 2009, volume 5717 of Lecture Notes in Computer Science, pages 383-390. Springer Berlin Heidelberg, 2009.

[2] R. Arkin. Behavior-based robotics (intelligent robotics and autonomous agents). the MIT Press, 1998.

[3] R. E. Bianchi and R. A. F. Romero. Sistema de navegação de robôs móveis autônomos para o transporte de documentos. Master's thesis, Universidade de SÃčo Paulo, http://www.scientificcommons.org/33797491, 2003.

[4] W. Burgard, A. B. Cremers, D. Fox, D. Hähnel, G. Lakemeyer, D. Schulz, W. Steiner, and S. Thrun. The interactive museum tour-guide robot. AAAI Proceedings, 1998. 
[5] J. Carbonell, O. Etzioni, Y. Gil, R. Joseph, C. Knoblock, S. Minton, and M. Veloso. Prodigy: an integrated architecture for planning and learning. SIGART Bull., 2:51-55, July 1991.

[6] C. I. Connolly and R. A. Grupen. The applications of harmonic functions to robotics. Journal of Robotic Systems, pages 931-946, 1993.

[7] E. Dijkstra. A note on two problems in connexion with graphs. Numerische mathematik, 1(1):269-271, 1959.

[8] A. Elfes. Using occupancy grids for mobile robot perception and navigation. Computer, 22:46-57, 1989.

[9] G. Faria. Uma Arquitetura de controle inteligente para múltiplos robôs. PhD thesis, Universidade de São Paulo, 2006.

[10] Y. Gakuhari, H. Takase, K. Hemeldan, and E. Hada. Delivery service robot using distributed acquisition, actuators and intelligence. IEEE/RSJ International Conference on Intelligent Robots and Systems (IROS), 3:2997 - 3002, 2004.

[11] B. P. Gerkey, R. T. Vaughan, and A. Howard. The player $\backslash$ stage project: Tools for multirobot and distributed sensor systems. Proceedings of the International Congress on Advanced Robotics, pages 317-323, June 2003.

[12] P. Hart, N. Nilsson, and B. Raphael. A formal basis for the heuristic determination of minimum cost paths. IEEE Transactions on Systems Science and Cybernetics, 4(2):100-107, 1968.

[13] J. R. Jang and C.-T. Sun. Neuro-fuzzy modeling and control. Proceedings fo the IEEE, 83:378-406, Mar. 1995.

[14] O. Khatib. Real-time obstacle avoidance for manipulators and mobile robots. IEEE International Conference on Robotics and Automation, pages 500-505, 1985.

[15] P. King and E. Mamdani. The application of fuzzy control systems to industrial processes. Automatica, 13(3):235-242, 1977.

[16] M. Lee, M. Tarokh, and M. Cross. Fuzzy logic decision making for multi-robot security systems. Artificial Intelligence Review, 34:177-194, 2010.

[17] R. Murphy. An introduction to AI robotics. MIT press, 2000.

[18] W. Pedrycz and F. Gomide. An Introduction to Fuzzy Sets: Analysis and Design. The MIT Press, 1998. 
[19] T. Reshmidevi, T. Eldho, and R. Jana. A gis-integrated fuzzy rule-based inference system for land suitability evaluation in agricultural watersheds. Agricultural Systems, 101(1 - 2):101 - 109, 2009.

[20] S. Russell, P. Norvig, J. Canny, J. Malik, and D. Edwards. Artificial intelligence: a modern approach, volume 2. Prentice hall Englewood Cliffs, NJ, 1995.

[21] Y. Sakagami, R. Watanabe, C. Aoyama, S. Matsunaga, N. Higaki, and K. Fujimura. The intelligent asimo: system overview and integration. In Intelligent Robots and Systems, 2002. IEEE/RSJ International Conference, volume 3, pages 2478-2483, 2002.

[22] B. M. Sathyaraj, L. Jain, A. Finn, and S. Drake. Multiple uavs path planning algorithms: a comparative study. Fuzzy Optimization and Decision Making, 7:257-267, 2008.

[23] R. G. Simmons, R. Goodwin, K. Z. Haigh, S. Koenig, J. O'Sullivan, and M. M. Veloso. Xavier: experience with a layered robot architecture. SIGART Bull., 8:22-33, December 1997.

[24] M. Sugeno and G. Kang. Fuzzy modelling and control of multilayer incinerator. Fuzzy Sets and Systems, 18:329-346, 1986.

[25] G. Tian, F. Wang, and J. Li. An improved best path methodology for mobile gis based on $\mathrm{a}^{*}$ algorithm. In International Conference on Artificial Intelligence, Management Science and Electronic Commerce, pages 2775 -2778, 2011.

[26] F. Velo, L. Baturone, S. Solano, and A. Barriga. Rapid design of fuzzy systems with xfuzzy. In Fuzzy Systems, 2003. FUZZ '03. The 12th IEEE International Conference on, volume 1, pages 342-347, may 2003.

[27] C.-J. Wu and T.-L. Lee. A fuzzy mechanism for action selection of soccer robots. Journal of Intelligent and Robotic Systems, 39:57-70, 2004.

[28] J. Yao, C. Lin, X. Xie, A. Wang, and C.-C. Hung. Path planning for virtual human motion using improved a* star algorithm. In International Conference on Information Technology: New Generations, pages $1154-1158,2010$.

[29] L. Zadeh. Fuzzy sets. Information and Control, (8):338-353, 1965. 